\title{
Quantitative Microbiological Evaluation of Salmonella Typhimurium Shed in Diarrhea, Loose, and Normal Stools of Infected Pigs
}

\author{
Tsuyoshi Tanaka ${ }^{1,2 *}$, Yasuo Imai ${ }^{1}$, Naosuke Kumagae ${ }^{2,3}$, Takashi Sasaki ${ }^{1}$, Narutoshi Ochiai ${ }^{1}$, \\ Katsuyoshi Uruno', Haruki Kitazawa ${ }^{2}$, Tadao Saito ${ }^{2}$, Shizuo Sato ${ }^{1}$ \\ ${ }^{1} \mathrm{JA}$ Zen-Noh (National Federation of Agricultural Co-operative Associations), Institute of Animal Health, \\ 7 Ohja-machi, Sakura, Chiba, Japan \\ ${ }^{2}$ Food and Feed Immunology Group, Laboratory of Animal Products Chemistry, Graduate School of Agricultural \\ Science, Tohoku University, Aobaku, Sendai, Miyagi, Japan \\ ${ }^{3}$ Scientific Feed Laboratory Co., Ltd., 7 Ohja-machi, Sakura, Chiba, Japan

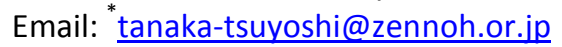

Received 11 March 2014; revised 6 April 2014; accepted 14 April 2014

Copyright (C) 2014 by authors and Scientific Research Publishing Inc.

This work is licensed under the Creative Commons Attribution International License (CC BY). http://creativecommons.org/licenses/by/4.0/

(c) $\underset{\mathrm{EY}}{\mathrm{BY}}$ Open Access

\begin{abstract}
Control of within-herd transmission of Salmonella is important for reducing the prevalence of this organism on pig farms and for preventing Salmonella-contamination of pork. At the farm level, understanding the within-herd transmission of Salmonella can lead to more effective control. Salmonella infection is dependent on the inoculation dose; hence, quantitative evaluation of Salmonella shed in feces would provide useful information for developing effective measures. In this study, to reproduce and evaluate the number of Salmonella shed in diarrhea, loose stools, and normal feces, weaned pigs were inoculated with $3.2 \times 10^{9}, 3.2 \times 10^{7}$, and $3.2 \times 10^{5}$ cfu of Salmonella Typhimurium, respectively. The number of $S$. Typhimurium shed in the feces peaked within 1 week post-inoculation in every group and the most amount of diarrhea and loose stools were observed within 2 weeks post-inoculation. Diarrhea occurred 10 times (six pigs), and loose stools were observed 25 times (11 pigs). The average concentration of $S$. Typhimurium shed in diarrhea, loose stools, and normal feces was $1.0 \times 10^{8}, 1.6 \times 10^{4}$, and $7.1 \times 10^{1} \mathrm{cfu} / \mathrm{g}$ feces, respectively. These data suggest that diarrhea and loose stools are significant sources of within-herd transmission of Salmonella. Moreover, as some of the normal feces contained $>1.0 \times 10^{6} \mathrm{cfu} / \mathrm{g}$ of $S$. Typhimurium, even normal feces could be a source of within-herd transmission of Salmonella. At Salmonella-positive farms, reduction of the amount of Salmonella shed even in normal feces would lead to better control of within-herd transmission of Salmonella. These data can contribute to the control of within-herd transmission of Salmonella, particularly during the weaning period.
\end{abstract}

*Corresponding author.

How to cite this paper: Tanaka, T., et al. (2014) Quantitative Microbiological Evaluation of Salmonella Typhimurium Shed in Diarrhea, Loose, and Normal Stools of Infected Pigs. Open Journal of Veterinary Medicine, 4, 58-66.

http://dx.doi.org/10.4236/ojvm.2014.44007 


\section{Keywords}

\section{Fecal Shedding, Quantitative Evaluation, Salmonella Typhimurium, Swine, Transmission}

\section{Introduction}

Salmonella infections of swine are important because Salmonella can cause salmonellosis in pigs and contaminate pork products and induce human food-borne disease [1]. It has been estimated that, in the 1990s, about 20\% of all human salmonellosis cases in the European Union were linked to the consumption of pork [2]. Implementing good biosecurity and operating at a high hygiene standard at the farm level itself is crucial for controlling Salmonella infections and for preventing human salmonellosis [3].

Controlling within-herd transmission of Salmonella leads to effective control of Salmonella infection on pig farms. To date, many studies have been performed to establish measures for controlling within-herd transmission of Salmonella [4]-[6]. A number of such studies attempted to mimic the situation at the herd level, in order to investigate the risk of transmission to naïve pigs when they are exposed to contaminated pens or infected pen mates [7] [8]. These studies clarified that the symptoms caused by a Salmonella infection and the amount of Salmonella shed in the feces are dependent on the inoculation dose [9]-[12]. However, most of these studies were focused on pigs in the fattening or finishing period, or pigs at abattoirs because the prevalence of Salmonella during these periods is directly related to Salmonella contamination of pork.

Salmonella Typhimurium is most frequently isolated serotype from pigs [13]. S. Typhimurium is a causative agent of pig enterocolitis and is the major agent of human food-borne disease [14] [15]. Pigs are susceptible to $S$. Typhimurium-induced diarrhea during the weaning period, and clinical salmonellosis is prevalent in these pigs at many farms in Japan and other countries [14]-[17]. Control of within-herd transmission of Salmonella during the weaning period reduces the prevalence of Salmonella during the fattening or finishing periods [18] [19]. One of the important targets for controlling the within-herd transmission of Salmonella during the weaning period is avoiding fecal deterioration caused by $S$. Typhimurium, as it is widely accepted that diarrhea samples contain large amounts of the organism. However, there have been insufficient scientific assessments of the amount of $S$. Typhimurium shed in diarrhea, loose stools, and normal feces.

Thus, the purpose of this study was to evaluate the amount of $S$. Typhimurium shed in diarrhea, loose stools, and normal feces after $S$. Typhimurium-inoculation of weaned pigs. These data can guide effective measures for controlling within-herd transmission of Salmonella during the weaning period, at the farm level.

\section{Materials and Methods}

This study was approved by the Animal Care and Use Committee of the Institute of Animal Health of JA ZenNoh (National Federation of Agricultural Co-Operative Associations) and all animal experiments were performed in accordance with the Guidelines for the Care and Use of Laboratory Animals established by this Committee.

\subsection{Experimental Design}

Fifteen 28-day-old specific pathogen-free (SPF) pigs were transported to our isolated facilities. Upon arrival, the pigs were assigned randomly to one of three groups $(n=5)$, namely, ST-9, ST-7, or ST-5; there were no weight differences among the groups. They were provided feed and water ad libitum.

During 7 days of acclimatization, fecal samples were collected on three occasions and serum samples were collected once to verify that the pigs were free of Salmonella, using culture tests and a commercial ELISA (SALMOTYPE ${ }^{\circledR}$ Pig Screen; Labor Diagnostik Leipzig, Leipzig, Germany) on the basis of previous reports [20] [21]. Based on these test results, all pigs were confirmed to be free from Salmonella.

The pigs were then inoculated with $3.2 \times 10^{5}$ (ST-5), $3.2 \times 10^{7}$ (ST-7), or $3.2 \times 10^{9}$ (ST-9) cfu of rifampicin-resistant $S$. Typhimurium strain 116 (ST116Rif ${ }^{r}$ ), respectively, to induce diarrhea and loose stools, as previously described [9]. Animals with severe salmonellosis, i.e., pigs presenting with severe dehydration and depression, were euthanized by exsanguination after deep anesthesia with sodium pentobarbital $(100 \mathrm{mg} / \mathrm{kg})$, and 
subjected to necropsy. At 39 DPI, surviving pigs were euthanized as discussed above and also subjected to necropsy.

\subsection{Inoculated Culture}

The S. Typhimurium strain, ST116Rif ${ }^{\mathrm{r}}$, used for inoculation in this study, was prepared as described previously [20]. Briefly, the inoculated strain ST116Rif ${ }^{\mathrm{r}}$ was cultured in $200 \mathrm{ml}$ of soybean-casein digest broth (Eiken Chemical Co., Ltd., Tokyo, Japan) for $5 \mathrm{~h}$ at $37^{\circ} \mathrm{C}$, with vigorous shaking. This culture was then diluted to the appropriate concentrations using sterile phosphate-buffered saline (PBS), and then mixed with an equal volume of $20 \%$ skimmed milk. The pigs were then individually inoculated with $10 \mathrm{ml}$ of this mixture via oral gavage. Specifically, each group of pigs was administered an oral dose of $10 \mathrm{ml}$ containing $3.2 \times 10^{4}$ (ST-5), 3.2 $\times 10^{6}(\mathrm{ST}-7)$, or $3.2 \times 10^{8}(\mathrm{ST}-9) \mathrm{cfu} / \mathrm{ml}$ of ST116Rif ${ }^{\mathrm{r}}$.

\subsection{Clinical Investigations and Collection of Samples}

All pigs were clinically examined on a regular basis. Clinical investigation included scoring of the condition of the feces. A fecal condition score of 0,1 , or 2 was assigned based on whether the feces appeared normal, loose, or diarrheal, respectively. Fecal samples were collected at $0,3,4,6,12,15,19,22,26,29,33$, and 36 days post-inoculation.

\subsection{Quantitative Culture of $S$. Typhimurium 116Rifr from Feces}

After collection, $1 \mathrm{~g}$ of the fecal samples was diluted serially in 10-fold increments in sterile PBS and plated on deoxycholate hydrogen sulfide agar (Eiken Chemical Co. Ltd.) containing up to $100 \mu \mathrm{g} / \mathrm{ml}$ of rifampicin (RFDHL). The samples were then incubated for $24 \mathrm{~h}$ at $37^{\circ} \mathrm{C}$, and the resulting colonies were counted. Next, enrichment and delayed secondary enrichment procedures were conducted. Briefly, $1 \mathrm{ml}$ of a $1 \mathrm{~g}$ fecal solution was diluted in $10 \mathrm{ml}$ of Hajna tetrathionate broth (Eiken Chemical, Co., Ltd.) followed by a 24-h incubation at $41.5^{\circ} \mathrm{C}$, or for an additional 7 days at room temperature, as a delayed secondary enrichment culture. After incubation, each culture was streaked on RFDHL plates. Enrichment procedures were discontinued if positive results were obtained before the experiment was completed.

\subsection{Qualitative Culture of $S$. Typhimurium 116Rifr at Necropsy}

At necropsy, $1 \mathrm{~g}$ each of liver, lung, spleen, tonsil, kidney, and ileocecal lymph node tissue, as well as of the contents of the cecum and the jejunum, were aseptically collected and placed in $10 \mathrm{ml}$ of Hajna tetrathionate broth. Samples were then incubated for $24 \mathrm{~h}$ at $41.5^{\circ} \mathrm{C}$, or for an additional 7 days at room temperature as a delayed secondary enrichment culture. After incubation, each culture was streaked on RFDHL plates.

\subsection{Statistical Analysis}

The number of ST116Rif ${ }^{\mathrm{r}}$ colonies was then log transformed, with zero interpreted as 1 organism/g of feces. A value of 100 organisms/g of feces was assigned to samples that were determined to be negative by direct plating methods, but which were positive by enrichment and secondary enrichment procedures [20] [21]. The total number of ST116Rif ${ }^{\mathrm{r}}$ was expressed as means \pm standard error. Statistical differences among groups were determined using a two-sided Student's $t$-test. In addition, the fecal condition scores obtained from the three groups were compared using the Mann-Whitney $U$ test. Differences with $P<0.05$ were considered significant.

\section{Results}

\subsection{Appearance of Salmonellosis and Fecal Conditions}

The pigs were maintained in a strictly controlled environment to ensure that the diarrhea was directly caused by $S$. Typhimurium inoculation. In group ST-9, four of five pigs were euthanized because of severe salmonellosis at 3, 4, 5, and 7 DPI (Figure 1). In groups ST-5 and ST-7, all of the pigs survived until the end of the experiment. All of the pigs in group ST-9 presented with diarrhea, except for one pig, which was euthanized due to severe salmonellosis before diarrhea could be confirmed. In group ST-7, two pigs had diarrhea and all of the 
Fig.1

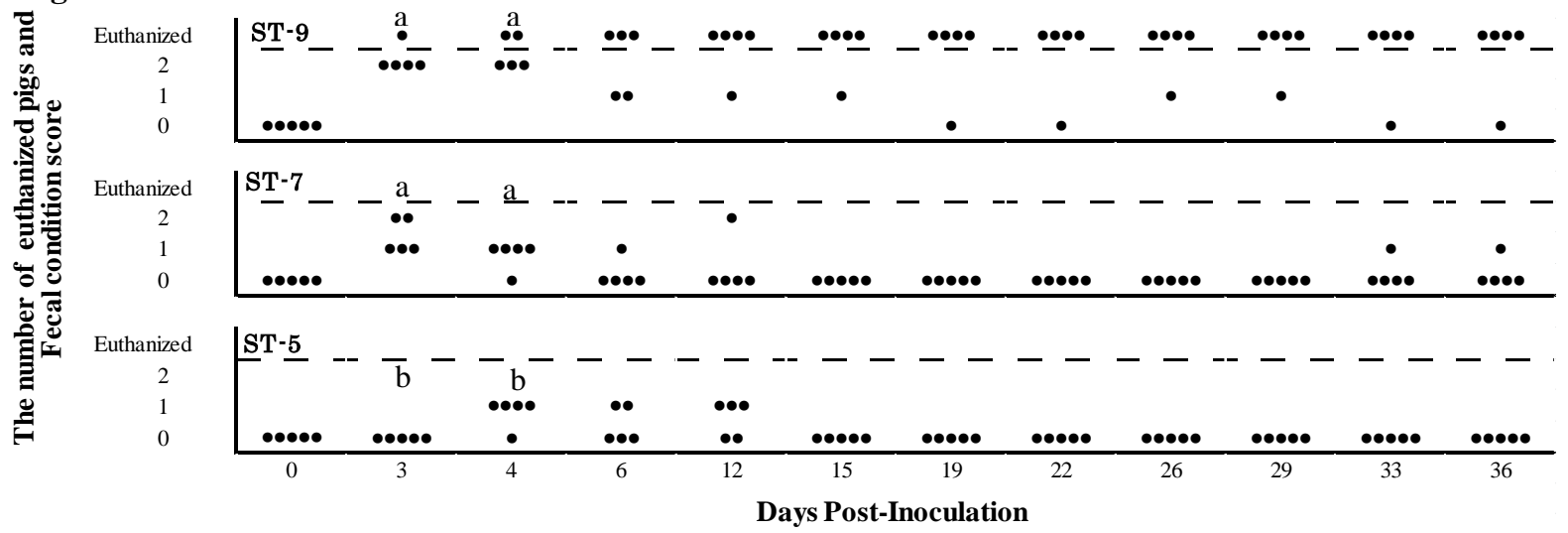

Figure 1. Severe salmonellosis, diarrhea, and loose stools induced by Salmonella Typhimurium 116Rif ${ }^{\mathrm{r}}$ inoculation. Each dot represents one pig. The pigs were each inoculated orally with $3.2 \times 10^{9}$ (ST-9), $3.2 \times 10^{7}$ (ST-7), or $3.2 \times 10^{5}$ (ST-5) cfu of $S$. Typhimurium 116Rifr. The pigs were euthanized if severe salmonellosis was observed. The condition of the feces produced by these pigs was scored. A fecal condition score of 0 indicated normal feces, 1 indicated loose stools, and 2 indicated diarrhea. Significant differences in the fecal condition score were observed between a and $b(P<0.05)$.

Fig.2

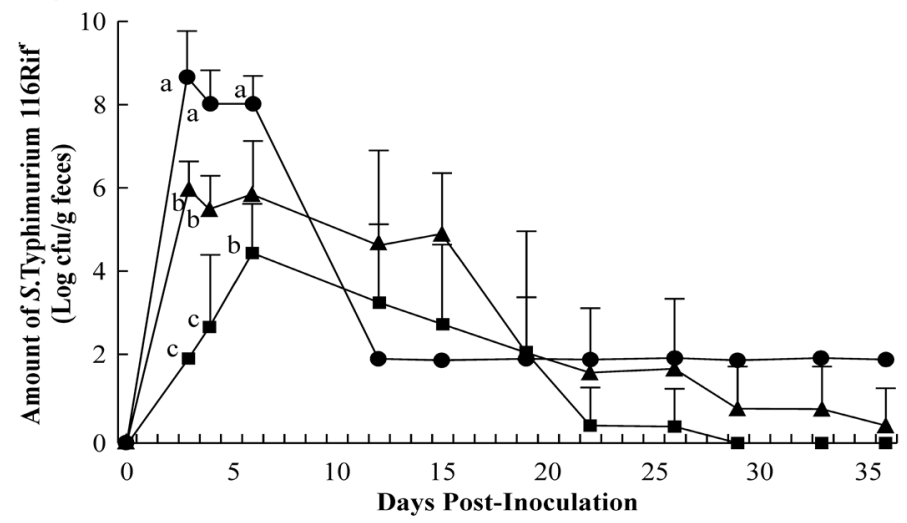

Figure 2. Amount of Salmonella Typhimurium 116Rif ${ }^{\mathrm{r}}$ shed in feces after inoculation with varying doses of $S$. Typhimurium 116Rif ${ }^{\mathrm{r}}$. The pigs were each inoculated orally with $3.2 \times 10^{9}, 3.2 \times 10^{7}$, or $3.2 \times$ $10^{5}$ cfu of $S$. Typhimurium 116 Rif $^{\mathrm{r}}$, as represented by a circle, triangle, and square, respectively. Significant differences in the amount of $S$. Typhimurium 116 Rif $^{r}$ shed in the feces were observed among the different groups (a, b, and c) $(P<0.05)$.

pigs produced loose stools. In group ST-5, none of the pigs had diarrhea, but four of five pigs produced loose stools. The fecal condition scores of ST-5 differed significantly from those of both ST-7 and ST-9, at both 3 and 4 DPI $(P<0.05)$. Most of the severe salmonellosis and diarrhea were observed within 7 DPI. Moreover, the fecal deterioration induced by $S$. Typhimurium infection was dependent on the inoculation dose.

\subsection{Correlation between $S$. Typhimurium 116Rifr Shed in Feces and the Inoculation Dose}

Fecal shedding of ST116Rif ${ }^{\mathrm{r}}$ peaked within 7 DPI in all groups (Figure 2), with the average peak of fecal ST116Rif ${ }^{\mathrm{r}}$ exceeding $1.0 \times 10^{8} \mathrm{cfu} / \mathrm{g}$ in group ST-9, $1.0 \times 10^{6} \mathrm{cfu} / \mathrm{g}$ in group ST-7, and $3.2 \times 10^{4} \mathrm{cfu} / \mathrm{g}$ in group ST-5. The ST116Rif ${ }^{\mathrm{r}}$ count in the stools of the pigs in the three groups differed significantly at 3 and 4 DPI $(P<$ 0.05), and the ST116Rif ${ }^{\mathrm{r}}$ count in the feces of pigs in groups ST-5 and ST-9 differed significantly at 6 DPI $(P<$ 0.05).

In group ST-9, four of five pigs were euthanized by 7 DPI, while the surviving pig continuously shed $1.0 \times$ $10^{2} \mathrm{cfu} / \mathrm{g}$ feces of ST116Rif ${ }^{\mathrm{r}}$; thus, the fecal ST116Rif ${ }^{\mathrm{r}}$ count declined immediately in group ST-9. In group 


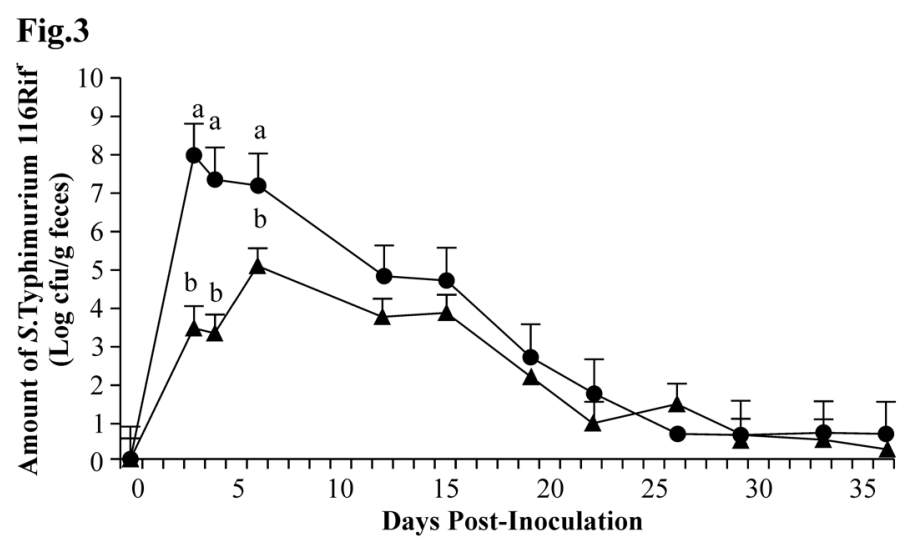

\begin{abstract}
Figure 3. Amount of Salmonella Typhimurium 116Rif ${ }^{\mathrm{r}}$ shed in the feces of diarrheal pigs and pigs producing loose stools, but not diarrhea, after inoculation with $S$. Typhimurium 116Rif ${ }^{\mathrm{r}}$. The amount of $S$. Typhimurium 116Rif ${ }^{\mathrm{r}}$ shed in feces after oral inoculation with $S$. Typhimurium 116Rif ${ }^{\mathrm{r}}$. The diarrhea group ( $\mathrm{n}=6$; circles) was composed of pigs observed to produce diarrhea, while the loose stools group $(\mathrm{n}=$ 7; triangles) was composed of pigs observed to produce loose stools, but not diarrhea. Significant differences were observed between a and b $(P<0.05)$.
\end{abstract}

ST-7, all of the pigs continuously shed ST116Rif in their feces until 15 DPI, while in group ST-5, all of the pigs continuously shed ST116Rif in their feces until 12 DPI. By the end of the experiment, fecal shedding of all of the pigs in group ST-7 and ST-5 had become intermittent.

\title{
3.3. Correlation between the Amounts of $S$. Typhimurium 116Rifr Shed in Feces by Pigs Producing Diarrhea and Those Producing Loose Stools
}

In this study, six pigs were observed to have diarrhea, i.e., four and two pigs from ST-9 and ST-7, respectively (diarrhea group). In the diarrhea group, three of six pigs survived until the end of the experiment. Moreover, seven pigs were observed to produce loose stools, but were not observed to have diarrhea; these included three and four pigs from ST-7 and ST-5, respectively (loose stools group). In this group, all pigs survived until the end of the experiment.

The amounts of ST116Rif ${ }^{\mathrm{r}}$ shed in feces of the pigs belonging to the two groups were compared (Figure 3). In the diarrhea group, the peak count of ST116Rif ${ }^{\mathrm{r}}$ shed in the feces was approximately $1.0 \times 10^{8} \mathrm{cfu} / \mathrm{g}$, while in the loose stools group, this count exceeded $1.0 \times 10^{5} \mathrm{cfu} / \mathrm{g}$. The counts were significantly different between the two groups at 3,4 , and 7 DPI (all $P<0.05$ ).

In both the diarrhea and loose stools groups, the pigs shed ST116Rif ${ }^{r}$ continuously until 15 DPI. In the diarrhea group, one of the three surviving pig continued to shed ST116Rif until the end of the experiment, but the other two pigs showed intermittent shedding at 19 and 26 DPI, respectively. All of the pigs in the loose stools group switched to intermittent ST116Rif ${ }^{\mathrm{r}}$ shedding at 19 (two pigs), 22 (three pigs), 29 (one pig), and 33 (one pig) DPI.

\subsection{Counts of $S$. Typhimurium 116Rifr Shed in Diarrhea, Loose Stools, and Normal Feces}

In this trial, diarrhea was observed 10 times: seven times in ST-9 and three times in ST-5 pigs (Figure 1). Loose stools were observed 25 times: six times in ST-9, ten times in ST-7, and nine times in ST-5 pigs. Normal feces were observed 92 times. The amounts of ST116Rif ${ }^{\mathrm{r}}$ shed in diarrhea $(\mathrm{n}=10)$, loose stool $(\mathrm{n}=25)$, and normal stool $(\mathrm{n}=92)$ samples were also compared.

The average counts of ST116Rif ${ }^{\mathrm{r}}$ shed in diarrhea, loose stools, and normal feces samples were $1.0 \times 10^{8}$ $\mathrm{cfu} / \mathrm{g}, 1.6 \times 10^{4} \mathrm{cfu} / \mathrm{g}$, and $7.1 \times 10^{1} \mathrm{cfu} / \mathrm{g}$, respectively (Table 1$)$; these counts differed significantly $(P<0.01)$. The counts of ST116Rif ${ }^{\mathrm{r}}$ shed in diarrhea ranged from $1.7 \times 10^{6}-5.3 \times 10^{9} \mathrm{cfu} / \mathrm{g}$. In loose stools, the highest ST116Rif ${ }^{\mathrm{r}}$ count was $3.2 \times 10^{8} \mathrm{cfu} / \mathrm{g}$. ST116Rif ${ }^{\mathrm{r}}$ was not detected in two loose stool samples, which were ob- 
Table 1. Salmonella Typhimurium 116 Rif $^{\mathrm{r}}$ shed in diarrhea, loose stools, and normal feces after inoculation with S. Typhimurium 116 Rifr'. $^{\mathrm{r}}$.

\begin{tabular}{|c|c|c|c|c|c|c|c|c|c|}
\hline \multicolumn{4}{|c|}{ Fecal Condition } & \multicolumn{3}{|c|}{ Diarrhea } & Loose stools & \multicolumn{2}{|c|}{ Normal feces } \\
\hline \multicolumn{4}{|c|}{ Not detected } & & & & 2 & \multicolumn{2}{|c|}{40} \\
\hline \multicolumn{10}{|c|}{$\begin{array}{l}\text { Count of ST116Rif }{ }^{\mathrm{r}} \text { shed in fecal samples } \\
\qquad(\log \mathrm{cfu} / \mathrm{g})\end{array}$} \\
\hline \multicolumn{4}{|c|}{2.0} & & & & 6 & \multicolumn{2}{|c|}{26} \\
\hline \multicolumn{4}{|c|}{$<2.0-\leq 3.0$} & & & & & \multicolumn{2}{|c|}{4} \\
\hline \multicolumn{4}{|c|}{$<3.0-\leq 4.0$} & & & & 3 & \multicolumn{2}{|c|}{7} \\
\hline \multicolumn{4}{|c|}{$<4.0-\leq 5.0$} & & & & 3 & \multicolumn{2}{|c|}{5} \\
\hline \multicolumn{4}{|c|}{$<5.0-\leq 6.0$} & & & & 5 & \multicolumn{2}{|c|}{5} \\
\hline \multicolumn{4}{|c|}{$<6.0-\leq 7.0$} & \multicolumn{2}{|r|}{$2^{* *}$} & & 4 & \multicolumn{2}{|c|}{5} \\
\hline \multicolumn{4}{|c|}{$<7.0-\leq 8.0$} & \multicolumn{2}{|r|}{2} & \multicolumn{2}{|r|}{1} & & \\
\hline \multicolumn{4}{|c|}{$<8.0-\leq 9.0$} & \multicolumn{2}{|r|}{4} & \multicolumn{2}{|r|}{1} & & \\
\hline \multicolumn{4}{|c|}{$<9.0-\leq 10.0$} & \multicolumn{2}{|r|}{2} & & & & \\
\hline \multicolumn{4}{|c|}{ Mean \pm S.E. $(\log c f u / g)$} & \multicolumn{2}{|c|}{$8.01 \pm 1.08$} & \multicolumn{2}{|r|}{$4.20 \pm 2.27$} & \multicolumn{2}{|c|}{$1.85 \pm 2.01$} \\
\hline \multicolumn{4}{|c|}{ Statistics $^{*}$} & \multicolumn{2}{|r|}{ a } & & b & & \\
\hline $\begin{array}{l}\text { "Significant diffe } \\
\text { ST116Rif'. After } \\
92 \text { times, respect }\end{array}$ & $\begin{array}{l}\text { aces were observ } \\
\text { ally inoculating } \mathrm{f} \\
\text { ly. The amounts }\end{array}$ & among & $\begin{array}{l}\text { e group } \\
\text { ying am }\end{array}$ & $\begin{array}{l}\text { indicated } \\
\text { nts of } S \text {. } \\
\text { Rif' shed }\end{array}$ & $\begin{array}{l}\text { y a, b, and } \\
\text { yphimuriun } \\
\text { n each type }\end{array}$ & $\begin{array}{l}\text { c; } P<0.01 \\
116 \text { Rif', d } \\
\text { f feces were }\end{array}$ & $\begin{array}{l}\text { The number of feca } \\
\text { hea, loose stools, an } \\
\text { alyzed. }\end{array}$ & $\begin{array}{l}\text { aples that co } \\
\text { mal feces we }\end{array}$ & $\begin{array}{l}\text { each num } \\
\text { ved } 10,2\end{array}$ \\
\hline & & Liver & Lung & Spleen & Tonsils & Kidney & $\begin{array}{l}\text { Ileocecal lymph } \\
\text { node }\end{array}$ & $\begin{array}{l}\text { Jejunum } \\
\text { content }\end{array}$ & $\begin{array}{l}\text { Cecum } \\
\text { content }\end{array}$ \\
\hline Surviving & Diarrhea & $0 / 3$ & $0 / 3$ & $0 / 3$ & $3 / 3$ & $0 / 3$ & $1 / 3$ & $3 / 3$ & $3 / 3$ \\
\hline pigs & Loose feces & $1 / 7$ & $1 / 7$ & $1 / 7$ & $6 / 7$ & $2 / 7$ & $3 / 7$ & $5 / 7$ & $3 / 7$ \\
\hline & Normal feces & $0 / 1$ & $0 / 1$ & $0 / 1$ & $0 / 1$ & $0 / 1$ & $0 / 1$ & $0 / 1$ & $0 / 1$ \\
\hline & Total & $1 / 11$ & $1 / 11$ & $1 / 11$ & $9 / 11$ & $2 / 11$ & $4 / 11$ & $8 / 11$ & $6 / 11$ \\
\hline Euthanized & Diarrhea & $3 / 3$ & $3 / 3$ & $3 / 3$ & $3 / 3$ & $3 / 3$ & $3 / 3$ & $3 / 3$ & $3 / 3$ \\
\hline pigs & Unknown & $1 / 1$ & $1 / 1$ & $1 / 1$ & $1 / 1$ & $1 / 1$ & $1 / 1$ & $1 / 1$ & $1 / 1$ \\
\hline & Total & $4 / 4$ & $4 / 4$ & $4 / 4$ & $4 / 4$ & $4 / 4$ & $4 / 4$ & $4 / 4$ & $4 / 4$ \\
\hline
\end{tabular}

The 10 pigs that survived the infection with $S$. Typhimurium were necropsied at 39 DPI. Another four pigs were euthanized during the monitoring period, because of severe salmonellosis. At necropsy, liver, lung, spleen, tonsil, kidney, ileocecal lymph node tissue samples, and samples of the contents of the jejunum and of the cecum were aseptically collected, and subjected to $S$. Typhimurium detection.

tained at 33 and 36 DPI. In normal feces, the highest ST116Rif ${ }^{\mathrm{r}}$ count was $6.7 \times 10^{6} \mathrm{cfu}^{\mathrm{g}}$; no ST116Rif ${ }^{\mathrm{r}}$ was detected in 40 normal fecal samples.

\subsection{Detection of $S$. Typhimurium 116Rifr at Necropsy}

The rate of detection of ST116Rif ${ }^{\mathrm{r}}$ in liver, lung, spleen, kidney, and ileocecal lymph node tissues was higher in the euthanized pigs that had shown severe salmonellosis than in those of the surviving pigs who were sacrificed at the end of the experiment (Table 2). In the surviving pigs, ST116Rif ${ }^{\mathrm{r}}$ was detected more frequently in tonsil samples and in the contents of the cecum and jejunum. However, the detection rate did not differ between pigs in the diarrhea and the loose stools groups.

\section{Discussion}

The purpose of this study was to evaluate the amount of $S$. Typhimurium shed in diarrhea, loose stools, and normal fecal samples produced by $S$. Typhimurium-inoculated weaned pigs. The amount of S. Typhimurium 
shed in diarrhea was significantly higher than that shed in loose and normal stools; the amount of $S$. Typhimurium shed in loose stools was also significantly higher than that shed in normal feces.

In this study, diarrhea, loose stools, and normal feces were produced by $S$. Typhimurium-inoculated weaned pigs, but the clinical symptoms and the amount of $S$. Typhimurium shed in the feces were dependent on the inoculation dose, as has been previously reported [9]-[12]. Our previous report, in which weaned pigs were inoculated with $S$. Typhimurium strain 116Rif $\mathrm{r}^{\mathrm{r}}$, the same strain as used in this study, showed similar results in terms of fecal deterioration and the amount of $S$. Typhimurium shed in feces in response to the inoculation dose [20].

Although some reports have evaluated the amount of Salmonella shed in feces, this is the first study that compared the actual count of Salmonella shed in feces according to the condition of the feces [22]-[24]. Previous reports involved the fattening or finishing period of pigs, during which pigs are not susceptible to diarrhea caused by $S$. Typhimurium.

Our study further supported existing data that showed that finishing pigs, naturally infected with $S$. Typhimurium, infrequently shed Salmonella at amounts $>1 \times 10^{6} \mathrm{cfu} / \mathrm{g}$ in normal feces [25]. Some of the normal feces in our study contained Salmonella at concentrations of $>1 \times 10^{6} \mathrm{cfu} / \mathrm{g}$ feces. These data suggest that during both weaning and fattening, pigs sometimes shed sufficient amounts of Salmonella to establish within-herd transmission, even when they produce normal feces.

Our study also showed that diarrheal stools contain high amounts of Salmonella; however, Casey et al. reported that diarrhea samples contained approximately $10^{3} \mathrm{cfu} / \mathrm{g}$ feces in experimentally infected weaned pigs [23]. The difference between our findings and those of Casey et al. may be due to variability in the pathogenicity of the strains of Salmonella used to establish infection. A previous study showed that Salmonella strains vary in their pathogenicity [26].

There have been some reports about the amount of Salmonella required to establish within-herd transmission. A previous study had shown that $5.0 \times 10^{2} \mathrm{cfu}$ of $S$. Typhimurium/g feces was sufficient to induce within-herd transmission [27]. Moreover, Boughton et al. reported that exposure of pigs to a contaminated environment containing $4 \times 10^{2}$ cfu of $S$. Typhimurium $/ 100 \mathrm{~cm}^{2}$ resulted in infection [28]. Additionally, pigs can become infected with $S$. Typhimurium when they are exposed to the organism at a concentration of $10^{3} \mathrm{cfu} / \mathrm{g}$ feces [29]. These findings suggest that diarrheal and loose stools induced by $S$. Typhimurium contain significant amounts of the organism and are significant sources of $S$. Typhimurium infection at pig farms. Furthermore, in this study, some of the normal feces contained $S$. Typhimurium in excess of $1 \times 10^{6} \mathrm{cfu} / \mathrm{g}$ feces, so that even normal feces could be a source of within-herd infection.

The pigs seen to produce diarrhea shed $S$. Typhimurium for longer periods than did pigs producing loose feces. The rate of detection of $S$. Typhimurium in the internal organs and contents of the digestive tract of pigs that had severe diarrhea was also higher than that in survived pigs observed to produce loose stools, but not diarrhea. Thus, pigs producing diarrhea or showing deterioration in fecal condition pose a high risk of becoming a source of within-herd Salmonella transmission, even when they have themselves recovered from diarrhea.

The hygienic measures taken to reduce the level of environmental contamination on pig farms constitute the basis for the control of Salmonella infection. This study revealed that diarrhea and loose stools are a significant source of Salmonella and pose a risk for within-herd transmission and moreover that some of the normal fecal samples contain sufficient amounts of Salmonella to establish within-herd transmission of Salmonella.

\section{References}

[1] Kuhn, K.G., Sørensen, G., Torpdahl, M., Kjeldsen, M.K., Jensen, T., Gubbesl, S., Bjeraqer, G.O., Wingstrand, A., Prosbo, L.J. and Ethelberg, S. (2010) A Long-Lasting Outbreak of Salmonella Typhimurium U323 Associated with Several Pork Products, Denmark, 2010. Epidemiology and Infection, 141, 260-268. http://dx.doi.org/10.1017/S0950268812000702

[2] Steinbach, G. and Hartung, M. (1999) Attempt to Estimate the Share of Human Salmonella infections, Which Are Attributable to Salmonella Originating from Swine. Berliner und Munchener Tierarztliche Wochenschrift, 112, 296-300.

[3] Dahl, J., Wingstrand, A., Nielsen, B. and Baggesen, D.L. (1997) Elimination of Salmonella typhimurium Infection by the Strategic Movement of Pigs. Veterinary Record, 140, 679-681. http://dx.doi.org/10.1136/vr.140.26.679

[4] Österberg, J., Susanna, L. and Wallgren, P. (2010) Direct and Indirect Transmission of Four Salmonella Enterica Serotypes in Pigs. Acta Veterinaria Scandinavica, 52, 30. http://dx.doi.org/10.1186/1751-0147-52-30

[5] Rostagno, M.H., Susan, D.E. and Donald Jr., C.L. (2011) Immunological, Physiological, and Behavioral Effects of Salmonella Enterica Carriage and Shedding in Experimentally Infected Finishing Pigs. Foodborne Pathogens and Disease, 


\section{8, 623-630. http://dx.doi.org/10.1089/fpd.2010.0735}

[6] Boughton, C., Egan, J., Kelly, G., Markey, B. and Leonard, N. (2007) Rapid Infection of Pigs Following Exposure to Environments Contaminated with Different Levels of Salmonella Typhimurium. Foodborne Pathogens and Disease, 4, 33-40. http://dx.doi.org/10.1089/fpd.2006.58

[7] Hurd, H.S., McKean, J.D., Griffith, R.W., Wesley, I.V. and Rostagno, M.H. (2002) Salmonella Enterica Infections in Market Swine with and without Transport and Holding. Applied and Environmental Microbiology, 68, 2376-2381. http://dx.doi.org/10.1128/AEM.68.5.2376-2381.2002

[8] Morgan, I.R., Krautil, F.L. and Craven, J.A. (1987) Effect of Time in Lairage on Caecal and Carcass Salmonella Contamination of Slaughter Pigs. Epidemiology and Infection, 98, 323-330. http://dx.doi.org/10.1017/S0950268800062075

[9] Loynachan, A.T. and Harris, D.L. (2005) Dose Determination for Acute Salmonella Infection in Pigs. Applied and Environmental Microbiology, 71, 2753-2755. http://dx.doi.org/10.1128/AEM.71.5.2753-2755.2005

[10] Boyen, F., Pasmans, F., Van Immerseel, F., Donné, E., Morgan, E., Ducatelle, R. and Haesebrouck, F. (2009) Porcine in Vitro and in Vivo Models to Assess the Virulence of Salmonella Enterica Serovar Typhimurium for Pigs. Laboratory animals, 43, 46-52. http://dx.doi.org/10.1258/la.2007.007084

[11] Österberg, J. and Wallgren, P. (2008) Effects of a Challenge Dose of Salmonella Typhimurium or Salmonella Yoruba on the Patterns of Excretion and Antibody Responses of Pigs. Veterinary Record, 162, 580-585. http://dx.doi.org/10.1136/vr.162.18.580

[12] Proux, K., Cariolet, R., Fravalo, P., Houdayer, C., Keranflech, A. and Madec, F. (2001) Contamination of Pigs by Noseto-Nose Contact or Airborne Transmission of Salmonella Typhimurium. Veterinary Research, 32, 591-600. http://dx.doi.org/10.1051/vetres:2001148

[13] Kishima, M., Uchida, I., Namimatsu, T., Osumi, T., Takahashi, S., Tanaka, K., Aoki, H., Matsuura, K. and Yamamoto, K. (2008) Nationwide Surveillance of Salmonella in the Faeces of Pigs in Japan. Zoonoses and Public Health, 55, 139144. http://dx.doi.org/10.1111/j.1863-2378.2007.01105.x

[14] Asai, T., Otagiri, Y., Osumi, T., Namimatsu, T., Hirai, H. and Sato, S. (2002) Isolation of Salmonella from Diarrheic Feces of Pigs. Journal of Veterinary Medical Science, 64, 159-160. http://dx.doi.org/10.1292/jvms.64.159

[15] O’Dea, M.A., Kabay, M.J., Carr, J., Wilcox, G.E. and Richards, R.B. (2011) Porcine Circovirus-Associated Disease in Weaner Pigs in Western Australia. Australian Veterinary Journal, 89, 122-130. http://dx.doi.org/10.1111/j.1751-0813.2010.00680.X

[16] Sakano, C., Morita, Y., Goto, K., Yokota, Y., Annaka, H., Fujita, M., Kobatake, S., Ishioka, T., Hoshino, T., Boonmar, S., Pulsrikarn, S., Nishina, A., Kozawa, K., Yamamoto, S. and Kimura, H. (2011) Prevalence and Genotype of Salmonella Choleraesuis in Gunma Prefecture, Japan. Thai Journal of Veterinary Medicine, 41, 321-326.

[17] Huang, T.M., Lin, T.L. and Wu, C.C. (2009) Serovar Distribution and Antimicrobial Susceptibility of Swine Salmonella Isolates from Clinically Ill Pigs in Diagnostic Submissions from Indiana in the United States. Letters in Applied Microbiology, 48, 331-336. http://dx.doi.org/10.1111/j.1472-765X.2008.02530.x

[18] Wales, A.D., Cook, A.J.C. and Davies, R.H. (2011) Producing Salmonella-Free Pigs: A Review Focusing on Interventions at Weaning. Veterinary Record, 168, 267-276. http://dx.doi.org/10.1136/vr.d1125

[19] Wilkins, W., Rajić, A., Waldner, C., McFall, M., Chow, E., Muckle, A. and Rosengren, L. (2010) Distribution of Salmonella Serovars in Breeding, Nursery, and Grow-to-Finish Pigs, and Risk Factors for Shedding in Ten Farrow-to-Finish Swine Farms in Alberta and Saskatchewan. Canadian Journal of Veterinary Research, 74, 81.

[20] Tanaka, T., Imai, Y., Kumagae, N. and Sato, S. (2010) The Effect of Feeding Lactic Acid to Salmonella Typhimurium Experimentally Infected Swine. Journal of Veterinary Medical Science, 72, 827-831. http://dx.doi.org/10.1292/jvms.09-0490

[21] Williams, R.D., Rollins, L.D., Pocurull, D.W., Selwyn, M. and Mercer, G.D. (1978) Effect of Feeding Chlortetracycline on the Reservoir of Salmonella Typhimurium in Experimentally Infected Swine. Antimicrobial Agents and Chemotherapy, 14, 710-719. http://dx.doi.org/10.1128/AAC.14.5.710

[22] Spieh, M.J., Shurson, G.C. and Johnston, L.J. (2008) Effects of Two Direct-Fed Microbials on the Ability of Pigs to Resist an Infection with Salmonella Enterica Serovar Typhimurium. Journal of Swine Health and Production, $16,27$.

[23] Casey, P.G., Gardiner, G.E., Case, G., Bradshaw, B., Lawlor, P.G., Lynch, P.B., Leonard, F.L., Stanton, C., Fos, R.P., Fitzgerald, G.F. and Hill, C. (2007) A Five-Strain Probiotic Combination Reduces Pathogen Shedding and Alleviates Disease Signs in Pigs Challenged with Salmonella Enterica Serovar Typhimurium. Applied and Environmental Microbiology, 73, 1858-1863. http://dx.doi.org/10.1128/AEM.01840-06

[24] Österberg, J., Lewerin, S.S. and Wallgren, P. (2009) Patterns of Excretion and Antibody Responses of Pigs Inoculated with Salmonella Derby and Salmonella Cubana. Veterinary Record, 165, 404-408. http://dx.doi.org/10.1136/vr.165.14.404 
[25] Pires, A.F., Funk, J.A., Lim, A. and Bolin, S.R. (2013) Enumeration of Salmonella in Feces of Naturally Infected Pigs. Foodborne Pathogens and Disease, 10, 933-937. http://dx.doi.org/10.1089/fpd.2013.1547

[26] Namimatsu, T., Asai, T., Osumi, T., Imai, Y. and Sato, S. (2006) Prevalence of the Virulence Plasmid in Salmonella Typhimurium Isolates from Pigs. Journal of Veterinary Medical Science, 68, 187-188. http://dx.doi.org/10.1292/jvms.68.187

[27] Fedorka-Cray, P.J., Whipp, S.C., Isaacson, R.E., Nord, N. and Lager, K. (1994) Transmission of Salmonella Typhimurium to Swine. Veterinary Microbiology, 41, 333-344. http://dx.doi.org/10.1016/0378-1135(94)90029-9

[28] Boughton, C., Egan, J., Kelly, G., Markey, B. and Leonard, N. (2007) Rapid Infection of Pigs Following Exposure to Environments Contaminated with Different Levels of Salmonella Typhimurium. Foodborne Pathogens and Disease, 4, 33-40. http://dx.doi.org/10.1089/fpd.2006.58

[29] Hurd, H.S., Gailey, J.K., McKean, J.D. and Rostagno, M.H. (2001) Experimental Rapid Infection in Market Swine Following Exposure to a Salmonella Contaminated Environment. Berliner und Münchener Tierärztliche Wochenschrift, 114, 382. 